\title{
Advanced Malignant Lung Disease: What the Specialist Can Offer
}

\author{
D. Gompelmann R. Eberhardt F.J.F. Herth \\ Pneumology and Respiratory Care Medicine, Thoraxklinik at the University of Heidelberg, Heidelberg, Germany
}

\section{Key Words}

Lung cancer • Malignant pleural effusion · Pericardial effusion - Superior vena cava syndrome

\begin{abstract}
Lung cancer is not only the most commonly diagnosed cancer worldwide, but it is still the leading cause for cancer-related death. The 5-year survival for lung cancer in Europe and in the USA is totally $16 \%$. Therefore, a palliative therapy regimen is required to control the disease and reduce symptoms with the objective of enhancing quality of life of lung cancer patients. In addition to chemotherapy that is still one of the most important pillars in the treatment of advanced lung cancer, further interventional strategies can be offered to improve a patient's quality of life. A locoregional tumour progression is frequently associated with malignant pleural effusion or pericardial effusion, central airway obstruction, tracheo-oesophageal fistula, severe haemoptysis or superior vena cava (SVC) syndrome threatening life and necessitating urgent palliation. Recurrent pleural effusion causing dyspnoea can be managed by pleurodesis, serial thoracocentesis or insertion of an indwelling catheter. Symptomatic malignant pericardial effusion often requires an urgent pericardiocentesis. Furthermore, surgical procedures, instillation of sclerosing agents or local chemotherapy should be considered in refractory pericardial effusion. The therapy regimen of central airway stenosis includes mechanical and
\end{abstract}

thermic endoscopic procedures providing rapid relief of symptoms. To prevent recurrence of airway obstruction, the insertion of a stent or palliative brachytherapy provide reestablishment of the patency of obstructed airways. Haemoptysis can be managed by bronchoscopic interventions as well as by arterial embolization or palliative thoracic radiotherapy. The therapy of SVC syndrome is dependent of histology. In small-cell lung cancer, chemotherapy is recommended. In non-small-cell lung cancer, stent insertion and/ or radiotherapy are the therapeutic pillars.

Copyright $\odot 2011$ S. Karger AG, Basel

Lung cancer is not only the most commonly diagnosed cancer worldwide with 1.35 million new cases per year, but despite the advances in therapy regimens it is also still the leading cause for cancer-related death with 1.18 million deaths annually. The 5-year survival for lung cancer in Europe and in the USA is totally 16\% [1]. It varies according to lung cancer stage and histology: the 5-year survival is totally $49 \%$ in case of local lung cancer, $16 \%$ in case of regional lymph node involvement and only $2 \%$ in case of metastatic disease. At the time of initial diagnosis, two thirds of all patients with lung cancer have distant metastases. Depending on histology, $50 \%$ of patients with non-small-cell lung cancer (NSCLC) present a metastatic spread at the time of diagnosis. The median survival of patients with NSCLC stage IV and also stage IIIB disease

\section{KARGER}

Fax +4161306 1234

E-Mail karger@karger.ch

www.karger.com
C 2011 S. Karger AG, Basel

0025-7931/11/0822-0111\$38.00/0

Accessible online at:

www.karger.com/res
Dr. Daniela Gompelmann

Thoraxklinik Heidelberg

Amalienstrasse 5

DE-69126 Heidelberg (Germany)

Tel. +496221 396 8087, E-Mail daniela.gompelmann@ thoraxklinik-heidelberg.de 


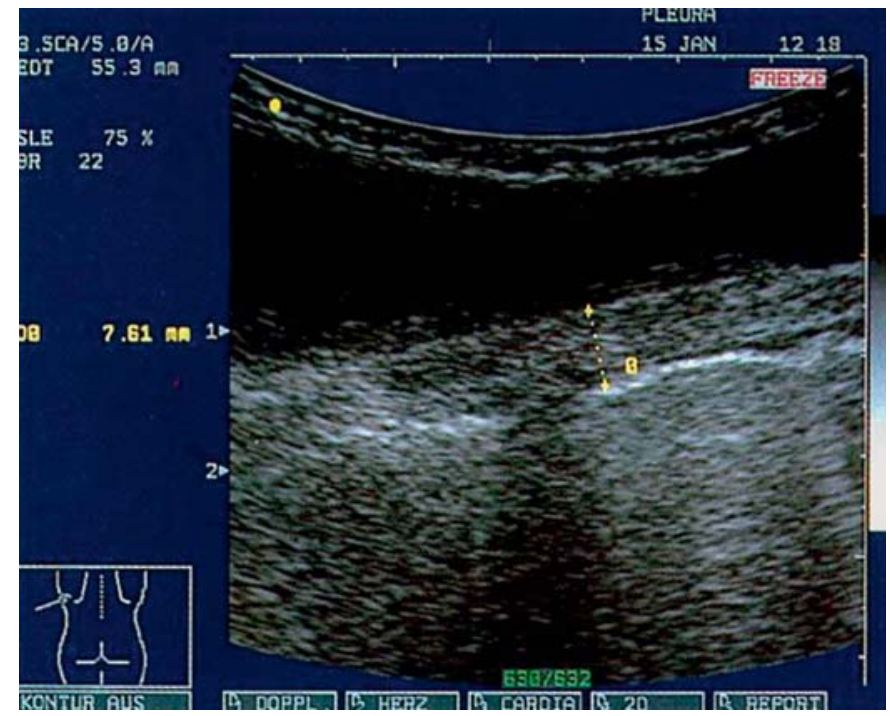

Fig. 1. Ultrasonography showing pleural thickening with pleural effusion.

who are not able to undergo curative radiotherapy is $8-$ 12 months. The presence of distant metastases in case of small-cell lung cancer (SCLC) is greater with $60-70 \%$. The median survival is $7-12$ months, and the proportion alive at 5 years is $2 \%$ [2].

These figures show that a palliative therapy regimen is needed to control the disease, reduce symptoms and enhance quality of life. One of the most important pillars in the therapy of advanced lung cancer is still chemotherapy to relieve cancer-related symptoms as well as prolong life. In NSCLC, platinum-based chemotherapy is recommended for patients with good performance status; single agents can be used in case of poor performance status [3]. Furthermore, molecularly targeted agents extend the spectrum of antineoplastic medication treatment. In extensive staged SCLC, a platinum-based combination with etoposide is the most commonly used treatment regimen in fit patients [3].

In addition to this neoplastic therapy, further interventional strategies can be offered to improve a patient's quality of life. A locoregional tumour progression frequently leads to local complications requiring an early diagnosis and subsequent rapid intervention. A malignant pleural effusion, malignant pericardial effusion, a central airway stenosis due to endobronchial tumour mass or extrabronchial tumour compression, tracheooesophageal fistula, severe haemoptysis or superior vena cava (SVC) syndrome threaten life and necessitate urgent palliation.
This manuscript reveals some interventional issues of palliative care in lung tumour patients including the management of locoregional complications.

\section{Pleural Effusion}

Lung cancer is the most common aetiology for malignant pleural effusion (MPE) accounting for 37\% of all malignant effusions [4]. MPE is defined as effusion in which malignant cells can be found. In the absence of cytological evidence of tumour cells, a pleural effusion is called paramalignant effusion in case of known malignant disease. According to the amount of pleural fluid, patients can be asymptomatic or present symptoms like dyspnoea due to restrictive ventilation, cough or pleuritic pain. Effusions can be diagnosed by chest X-ray showing blunting of costophrenic angles. In $10 \%$ of patients with MPE, a complete opacification of the hemithorax due to massive pleural effusion can be detected [5]. Other diagnostic methods to identify previously unrecognized small pleural effusions are ultrasonography with a sensitivity approaching 100\% [1] (fig. 1) or computed tomography. Depending on symptoms, treatment is required. Thoracentesis is the initial investigation in the management of MPE to determine if the patient benefits from the removal of pleural fluid. Up to $50 \%$ of the patients do not have beneficial effects from thoracentesis [4], because the breathlessness is caused by further concomitant morbidities like trapped lung by carcinomatous lymphangitis or atelectasis by bronchial obstruction. In case of relief of symptoms following evacuation of pleural fluid, thoracentesis can be repeated in refractory effusion for palliation of dyspnoea especially in patients with poor performance status and limited survival of weeks to months. However, the risk of infection by recurrent thoracenteses and discomfort for the patient should be considered, and a definitive intervention to prevent fluid reaccumulation should be performed. Two procedures can be differentiated: on the one hand pleurodesis by drainage, medical thoracoscopy/pleuroscopy or video-assisted thoracic surgery (VATS) can be considered to achieve fusion between the pleurae and thus stop fluid re-accumulation; on the other hand, an indwelling catheter can be inserted to provide outpatient management of recurrent pleural effusion.

\section{Pleurodesis}

Pleurodesis should be offered to patients with a good performance status for a local treatment of refractory 
MPE [6]. To achieve fusion between the pleurae, mechanical abrasion, partial resection of pleura or chemical pleurodesis can be performed whereby the latter procedure is the commonly used method. This procedure consists of insertion of a sclerosing agent inducing pleural inflammation and thus leading to adhesion of visceral and parietal pleura. Thereby, talc is the most commonly used sclerosing agent worldwide. It can be instilled through a chest tube or it can be insufflated as a dry powder during VATS or medical thoracoscopy. The instillation through a chest tube is a very easy, effective and widely available procedure that provides the management of recurrent MPE also in patients with a poor general condition. Thereby the success rate of preventing reaccumulation of pleural fluid is higher in patients with daily drainage of less than $200 \mathrm{ml}$ before talc slurry is instilled and if the time period between the diagnosis of MPE and onset of chest tube drainage is less than 30 days [7]. The talc poudrage by VATS or thoracoscopy/pleuroscopy however consists of insufflation of dry talc to all parts of the pleural surface under visual control. The VATS performed by the surgeon in the operating room is a minimally invasive thoracic surgery requiring singlelung ventilation under general anaesthesia. Through 1-3 entry sites, the whole pleural surface can be explored and the talc poudrage can be performed under direct visual guidance. On the other hand, a medical thoracoscopy/ pleuroscopy can be performed under local anaesthesia or conscious sedation in an endoscopy unit, using rigid or semi-flexible instruments through a single port. As no general anaesthesia is required, this procedure provides treatment of patients with very poor performance status who are not able to undergo general anaesthesia and single-lung ventilation. The advantage for talc poudrage compared to talc slurry is the complete evacuation of pleura fluid prior to pleurodesis and the homogeneous drug distribution. Furthermore, VATS or thoracoscopy/ pleuroscopy provides identification of trapped lung and evaluation of intrapleural tumour spread (fig. 2) [6]. Several studies comparing the effectiveness and safety of talc slurry through a chest tube thoracostomy with thoracoscopic talc poudrage revealed controversial results. In one prospective multicentre study [8], 501 patients with MPE were randomized to 2 study arms: one group of patients $(\mathrm{n}=242)$ underwent thoracoscopy with talc insufflation, and the other group of patients $(n=240)$ was treated by thoracostomy with talc slurry. There was no clear difference in efficacy between these two study arms (thoracoscopy with talc insufflation $78 \%$ vs. thoracostomy with talc slurry 71\%). However, the subgroup of patients with

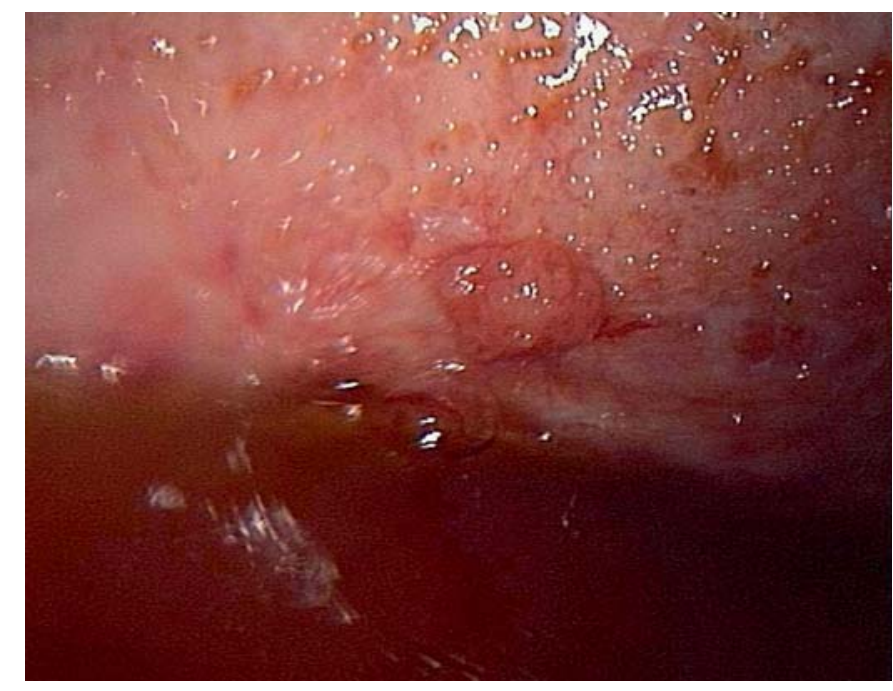

Fig. 2. Medical thoracoscopic view into the pleural cavity showing pleural carcinosis with tumour nodules covering the pleura.

primary lung or breast cancer had a higher success rate with thoracoscopy with talc insufflation than with thoracostomy with talc slurry ( 82 vs. $67 \%$ ). Another prospective, but non-randomized trial with 109 patients undergoing an interpleural talc insertion after lung re-expansion after drainage of MPE either by thoracoscopic talc poudrage $(\mathrm{n}=72)$ or by talc slurry through a chest tube $(\mathrm{n}=37)$ confirmed an effective response in preventing recurrence of MPE [9]. Nevertheless, thoracoscopic talc poudrage was significantly more effective than talc slurry through the drainage. In the talc poudrage group, $87.5 \%$ of the patients had an immediate successful pleurodesis; in the talc slurry group, $73 \%$ achieved rapid pleurodesis. Both methods were safe but in case of talc slurry, a higher incidence of thoracic pain could be revealed during the procedure. In a systematic review of 46 randomized clinical trials referring to the management of MPE published in 2006, the evaluation of the different methods of talc instillation revealed thoracoscopic talc insufflation as superior. Nevertheless, this comparison is based only on 2 small studies. Therefore, if thoracoscopy is unavailable, bedside talc pleurodesis has a high success rate and should be the step to be considered [10].

\section{Indwelling Catheter}

If a pleurodesis is not possible due to a poor general condition of the patient with a very short life expectancy or incomplete lung re-expansion after evacuation of pleural fluid, an indwelling catheter can be inserted for intermittent drainage allowing outpatient management. This 
tunnelled small catheter remains in the pleural cavity and can be connected intermittently to a vacuum bottle. This method prevents recurrent hospitalization. In a prospective study [11] referring to the effectiveness and safety of the indwelling catheter PleurX, 51 patients with MPE who were unsuitable for pleurodesis were enrolled. One day after implantation, $71 \%$ of the patients could be discharged home. Early complications like pneumothorax, subcutaneous emphysema and pain were observed in $8 \%$ of the patients. In $14 \%$ of all patients, late catheterrelated complications like infection or catheter dislodgement were discovered. Fusion between the pleurae with subsequent catheter removal could be achieved in 11 out of the 51 patients (21\%). In summary, the indwelling catheter seems to be a safe option for the management of MPE in the outpatient management.

In summary, a symptomatic MPE requires a multistep management with the goal of palliating the symptoms and preventing fluid recurrence. Therapeutic modalities include pleurodesis, serial thoracentesis or insertion of an indwelling catheter. In patients presenting with a good performance status, pleurodesis should be performed under visual guidance by video-assisted thoracoscopy or by pleuroscopy. A talc surry should be offered to patients who are not able to undergo the latter procedures. For patients in a very poor general condition and with a very short life expectancy or incomplete lung re-expansion after evacuation of pleural fluid, an indwelling catheter providing outpatient management or serial thoracentesis should be considered.

\section{Pericardial Effusion}

In autopsy series, the prevalence of pericardial and myocardial metastases is $21 \%$ in patients with malignant diseases. Of the underlying primary tumours, $36.5 \%$ are lung cancer [12]. The pericardial tumour spread causes typically malignant pericardial effusion (MPE). However, autopsy series overestimate the clinical problem of pericardial effusion, because microscopic metastases with only less effusion are without clinical relevance [13]. Depending on the amount of fluid, patients are asymptomatic or present symptoms of cardiovascular decompensation. The storage of pericardial fluid with cardiac impairment is known as cardiac tamponade - a lifethreatening syndrome requiring urgent intervention. Dyspnoea, tachypnoea, tachycardia, hypotension, pulsus paradoxus and jugular vein distension are frequent features in case of cardiac tamponade. Diagnosis is mainly made by echocardiography, but pericardial effusion can also be evident in chest X-ray and computed tomography. The electrocardiogram can also reveal typical signs of pericardial effusion.

The first step in the management of pericardial effusion is the percutaneous drainage under echocardiographic guidance [13] providing a rapid relief of symptoms. This intervention is tolerated with low morbidity and mortality also by patients with a poor performance status [14]. To achieve complete apposition of the parietal and visceral pericardium, an indwelling pericardial catheter can also be inserted for several days for extended drainage [15]. The efficacy of the insertion of these indwelling pericardial catheters has already been documented by Kopecky et al. [16] in 1986 and was confirmed by following studies [14].

The recurrence rate following pericardiocentesis or extended catheter drainage is however up to $40 \%$ without further treatment. Thereby, different approaches are available to minimize the risk of recurrence: surgical treatment, sclerosing therapy and local chemotherapy.

\section{Surgical Procedures}

The most common surgical interventions are pericardiotomy using a subxiphoid approach or the creation of a pleuropericardial window by video-assisted thoracoscopy or left thoracotomy. The principle of these approaches consists of creating a persistent communication through which fluid is drained and of inducing an inflammatory reaction leading to an adhesion of the two pericardial layers [13]. The advantage of the surgical procedures over the single pericardiocentesis was documented by several studies. A retrospective analysis published in 2008 regarding the treatment of MPE confirmed the efficacy and safety of balloon pericardiotomy as initial management. The re-accumulation rate following balloon pericardiotomy was $7.4 \%$ compared to $14.3 \%$ following simple aspiration [17].

\section{Sclerosing Therapy}

Sclerosing therapy following drainage provides another option to manage recurrent pericardial effusion by promoting adhesion between the parietal and visceral pericardium. 'Pure' sclerosing agents and 'cytotoxic' agents can be distinguished. The first agents used for slerosing therapy were 'pure' sclerosing agents like tetracycline or doxycycline. The efficacy could be documented in several studies $[18,19]$, but these agents were accompanied by a lot of adverse events like chest pain or fever. Therefore, 'cytotoxic' sclerosing agents that had already 
been used in pleural effusions were also tested in pericardial effusions. Several studies evaluating the sclerosing effect of bleomycin in MPE documented the effectiveness of this agent, its superiority to 'pure' slerosing agents due to less morbidity [20] and its superior efficacy to pericardial drainage alone [21]. Another 'cytotoxic' slerosing agent that can be used to prevent recurrent pericardial effusion is thiotepa (triethylene thiophosphoramide).

\section{Local Chemotherapy}

The goal of intrapericardial chemotherapy is regression of pericardial and myocardial metastases by a high local concentration of the antineoplastic drug. Carboplatin [22], cisplatin [23] or mitoxantrone [12] for example can be used for intrapericardial instillation to achieve control of refractory pericardial effusion.

In conclusion, symptomatic MPE often requires urgent intervention. In a life-threatening situation due to cardiac tamponade, a pericardiocentesis provides rapid relief of symptoms. In cases of refractory pericardial effusion, surgical procedures, instillation of slerosing agents or local chemotherapy are available. Up to date, there has been no preference for one of these strategies due to lack of randomized controlled studies. The therapy regimen should be orientated to the performance status of the patient.

\section{Central Airway Obstruction}

Central airway obstruction $(\mathrm{CAO})$ is defined as a reduced lumen at the level of the trachea, main carina and the main bronchi. Most central airway stenoses are caused by lung cancer [24] due to intraluminal tumour growth (fig. 3) or extraluminal compression. Symptoms include wheezing, cough, stridor, haemoptysis or chest pain depending on the loss of airway diameter. They develop rapidly and with increasing airway obstruction represent an acutely life-threatening state requiring urgent intervention. If the trachea is narrowed to less than $8 \mathrm{~mm}$, the patients will experience dyspnoea; a diameter less than $5 \mathrm{~mm}$ will result in respiratory distress. Furthermore, atelectasis and poststenotic pneumonia can result from airway obstruction.

There are different endoscopic procedures alleviating the acute obstruction: laser-assisted resection, electrocautery, cryodebridement, implantation of airway stents and intraluminal brachytherapy.

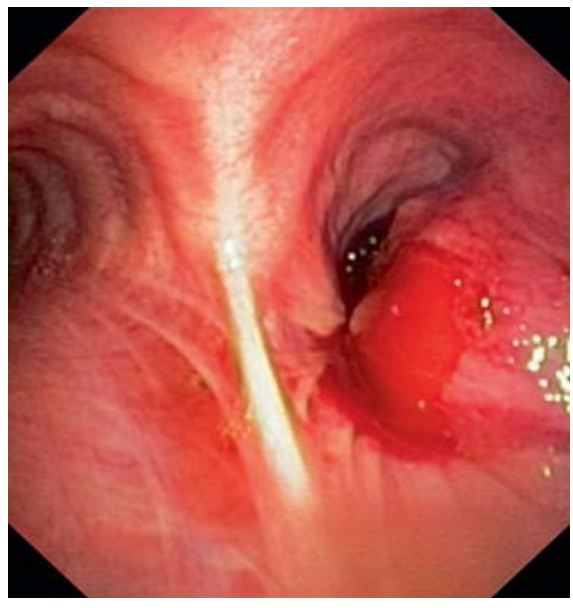

Fig. 3. Bronchoscopic view: endobronchial tumour growth in the right main bronchus.

\section{Laser}

Endoscopic laser (light amplification of stimulated emission of radiation) producing a beam of monochromatic, coherent light that can induce tissue vaporization, coagulation, haemostasis and necrosis [25] is one of the most important methods for bronchial debulking in case of endobronchial tumour growth [26]. Thereby, the Nd:YAG laser (neodymium:yttrium, aluminium and garnet) is one of the most commonly used lasers in the airways. Contact and non-contact fibres are available for YAG laser. The combination of laser with mechanical coring that is often used to achieve more rapid debulking is known as laser-assisted resection. First, the lesion is coagulated by laser followed by mechanical resection [26]. Risks of laser therapy are hypoxia, bleeding, perforation and fire [26]. To prevent the latter risk, the reduction of the inspiratory oxygen fraction to $50 \%$ prior to laser therapy is required. A study referring to the treatment of CAO with laser, stents and intraluminal brachytherapy in 2,008 patients demonstrated that in $93 \%$ of all patients undergoing laser resection, an immediate patency of the airway with consequent improvement of quality of life could be obtained [27]. The treatment was less successful in more peripheral lesions or with associated extrinsic airway compression. Zaric et al. [28] confirmed the beneficial effects of Nd:YAG laser concerning the symptom control in lung cancer patients. Furthermore, the use of laser can be combined with other methods, e.g. with implantation of stents, endoluminal brachy therapy or external beam radiation therapy (EBRT) preventing rapid reobstruction [26]. 


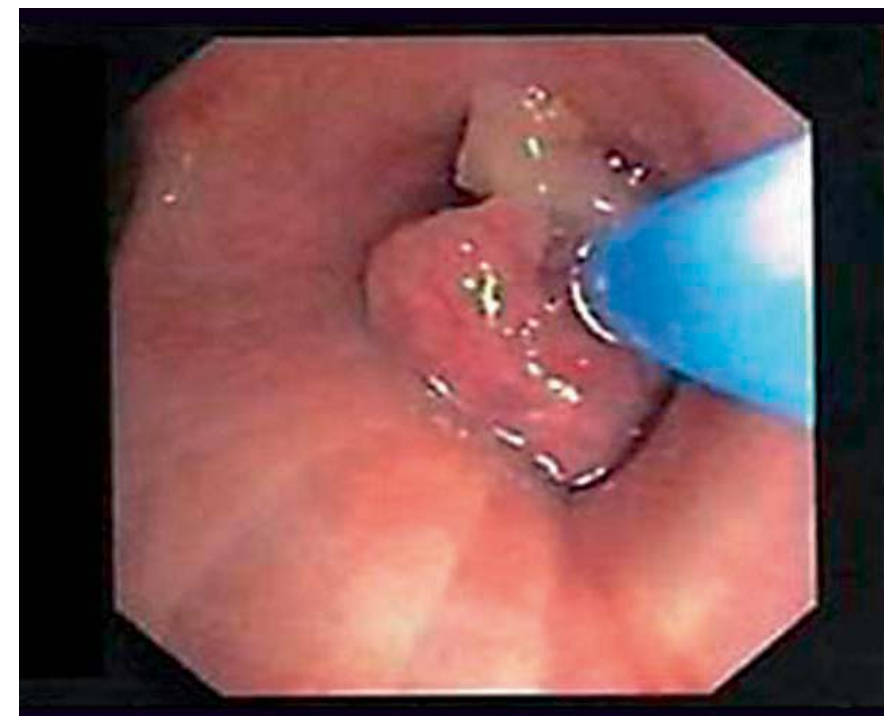

Fig. 4. Tumour debulking by cryotherapy.

\section{Electrocautery and Cryotherapy}

Alternatives to laser therapy to alleviate airway obstruction are electrocautery or cryotherapy [29]. Electrocautery induces coagulation and vaporization leading to tissue destruction. Argon plasma coagulation (APC) which provides a non-contact mode of electrocautery is very similar to the conventional electrocautery technique but using argon gas to conduct the electrical current [30]. The advantage of APC compared to electrocautery is its acute superficial tissue destruction with subsequent quick haemostasis. Therefore, APC can especially be used not only in case of airway obstruction, but also to manage severe haemoptysis due to endoluminal tumour growth. The beneficial effects of APC for treatment of both haemoptysis and airway stenosis was confirmed in a retrospective study published in 2001 [31], in which 60 patients were enrolled. All patients with haemoptysis experienced a haemostasis immediately after APC. Furthermore, APC leads to a decrease in endoluminal airway obstruction with subsequent improvement of symptoms.

Cryotherapy provides another technique for the management of tracheobronchial stenosis. Through hypothermic cellular crystallization and microthrombosis, a necrosis of tumour tissue is induced [30]. To achieve effective cryodebridement in $\mathrm{CAO}$, the distal probe tip with a temperature of $-89^{\circ} \mathrm{C}$ is placed in direct contact with the endoluminal tumour and pulled back after having frozen the tumour (fig. 4). In 2010, Schuhmann et al. [32] reported the results of a prospective study on endobron- chial tumour debulking with a flexible cryoprobe in 225 patients. In $91.1 \%$ of the patients, a cryorecanalization was successfully achieved. A mild or moderate bleeding occurred in $12 \%$ of the patients. Therefore, the use of cryoprobes seems to be a successful and safe procedure for tumour debulking. However, cryotherapy compared to laser or APC has a less immediate bleeding control, which has to be considered.

\section{Stent Implantation}

Besides mechanical and thermal endoscopic procedures, stents have been available for over 20 years in the treatment of central airway stenosis. Tracheobronchial stents are implanted to re-establish patency of extraluminal compressed airways as well as to stabilize airway patency after endoscopic removal of endoluminal obstruction resulting from malignant processes [33]. On the one hand, the insertion of airway stents provides treatment in the terminal care of patients, on the other hand tracheobronchial stenting has the function of a bridge until further tumour-specific treatment can be performed. In case of severe obstruction of the trachea or main bronchi, a straight stent can be implanted. There are many different stents, but in general, they can be divided into 2 groups, depending on the material: silicone stents and self-expandable metallic stents (SEMS). The most commonly used silicone stent is the Dumon stent [33] that has become the 'gold standard' for the treatment of malignant stenosis. These stents are covered with little studs on their surface to prevent stent dislocation. The inner surface is very smooth so that problems with incrusted secretions are rare. The Ultraflex stent, one of the SEMS, is made of nitinol that can adapt very well to kinked airways. Two types of Ultraflex stents are available: covered and noncovered stents. Another straight SEMS is the Nanjing Eco stent, also made of nitinol wire, that has an expansion force that is twice as high as the Ultraflex stent so that predilatation is not so commonly required as using the Ultraflex stent to accomplish good airway patency. Several studies regarding the use of these different straight stents confirm the efficacy of all of them in relieving respiratory symptoms [34-36].

In case of main carinal obstruction, the use of straight stents is limited due to the Y-shaped anatomy of the carina. Therefore, Y-shaped stents consisting of the tracheal body and 2 bronchial limbs are necessary to treat airway obstructions involving the lower trachea, main carina and proximal mainstem bronchi. In some case reports, the use of Y-shaped stents for airway stenoses around the carina between the right upper lobe bronchus 
and the bronchus intermedius is also described, but this technique has to be reviewed in further studies [37]. Yshaped polymer stents, hybrid stents and SEMS can be differentiated. The most commonly used polymer stent is the silicone Dumon Y stent [38] whose structure is comparable to the straight Dumon stent. The dynamic Ruesch stent [39], a Y-shaped hybrid stent, is a silicone stent with a flexible membrane in the position of the posterior tracheal wall, hence improving the action of coughing and reducing the amount of secretion retention. One Yshaped SEMS is the bifurcated Nanjing stent that is also known as $\mathrm{Y}$ carina ecostent. This is a woven metallic stent consisting of highly elastic nitinol wire. The first clinical results of the implantation of Y-shaped SEMS were published by Yang et al. [40]. In 2007, 5 patients with a complex tracheobronchial stenosis due to lung cancer or oesophageal cancer were successfully treated with these metallic stents. All patients had immediate relief of respiratory symptoms of dyspnoea or cough. Most studies of the efficacy of endobronchial Y-shaped stent placement have had impressive results confirming the benefits of stent placement in case of severe tracheobronchial stenosis [38-40].

Despite the benefit of the airway stents, there are some possible stent-related problems. One of the most commonly reported side effects is mucostasis due to the lack of mucociliary clearance inside polymer and covered stents, and due to the inability to cough efficiently. Furthermore, colonization with bacteria and fungi of this mucus layer is a common event associated with halitosis and infection. A review in 2009 including 23 articles involving 501 patients with airway stents revealed that $19 \%$ out of the 501 stented patients experienced stent-associated respiratory infection. Pneumonia was the most common type (47\%), followed by bronchial infection (24\%), cavitary pneumonia/lung abscess and intraluminal fungus ball [41]. The best measure to prevent mucostasis is a regular inhalation with saline. Furthermore, anti-inflammatory topical drugs can be used and are probably more useful than mucolytics. Another stent-related complication is the development of granulation tissue due to the high localized pressure of the stents on the mucosa [33]. To reduce the tissue hyperproliferation, the airway stents should fit tight to avoid dynamic friction without a high pressure on the mucosa that would impair microcirculation. Another complication is the stent luminal narrowing due to stent invasion by adjacent tumour tissue [33]. A restenosis due to occlusion by malignant tissue or granulation tissue formation requires mechanical recanalization, cryotherapy or argon beamer coagulation to restore airway patency. In addition, internal or external radiation therapy afterwards can prevent the development of granulation tissue. Stent migration and stent fracture are further stent-related problems that should be considered as serious risk factors.

\section{Brachytherapy}

Endobronchial brachytherapy provides another option for the treatment of CAO consisting of radiation delivered internally through a bronchoscopically inserted wire [25]. Brachytherapy should be considered as a part of a multimodality therapy to restore the patency of narrowed central airways. The effects of brachytherapy are delayed so that this technique is not suitable in life-threatening cases of $\mathrm{CAO}$ [42].

There are 2 different techniques to facilitate radiation delivery in the lung: on the one hand interstitial permanent volume implantation, on the other hand temporary endoluminal or interstitial implants [42]. Furthermore, a high-dose-rate (HDR) brachytherapy lasting some minutes and a low-dose-rate brachytherapy can be differentiated, whereby the HDR brachytherapy is the preferred method. The most common source is ${ }^{192}$ Ir with a high specific activity.

The endobronchial brachytherapy is performed under conscious sedation by a flexible bronchoscope [42]. First, a polyethylene catheter is endoscopically inserted under fluoroscopic guidance to verify the correct position in the airway close to the tumour lesion. After a second control of the right position with a dummy seed probe, the applicator is introduced and connected to the ${ }^{192} \mathrm{Ir}$ remote afterloading unit. Several studies confirmed the efficacy of brachytherapy in the palliation of local symptoms like dyspnoea, haemoptysis and cough $[43,44]$.

A recently published retrospective study revealed the efficacy and toxicity of HDR brachytherapy [45]. This study includes 226 patients with endoluminal NSCLC without extrabronchial spread but contraindications for EBRT or surgery. A complete local response was obtained in $93.6 \%$ at 3 months in all patients. Disease-free survival was $68 \%$ at 2 years and $50 \%$ at 5 years. In $10.4 \%$ of all patients, brachytherapy had to be interrupted due to acute respiratory insufficiency, bronchial ulceration, mucositis, progression and pneumothorax.

Despite these good results of HDR brachytherapy, it should not be recommended as the only therapeutic option to palliate local symptoms in previously untreated patients. A review [46] including 29 trials regarding palliative radiotherapy demonstrated that EBRT alone is preferable to HDR brachytherapy in previously untreated 


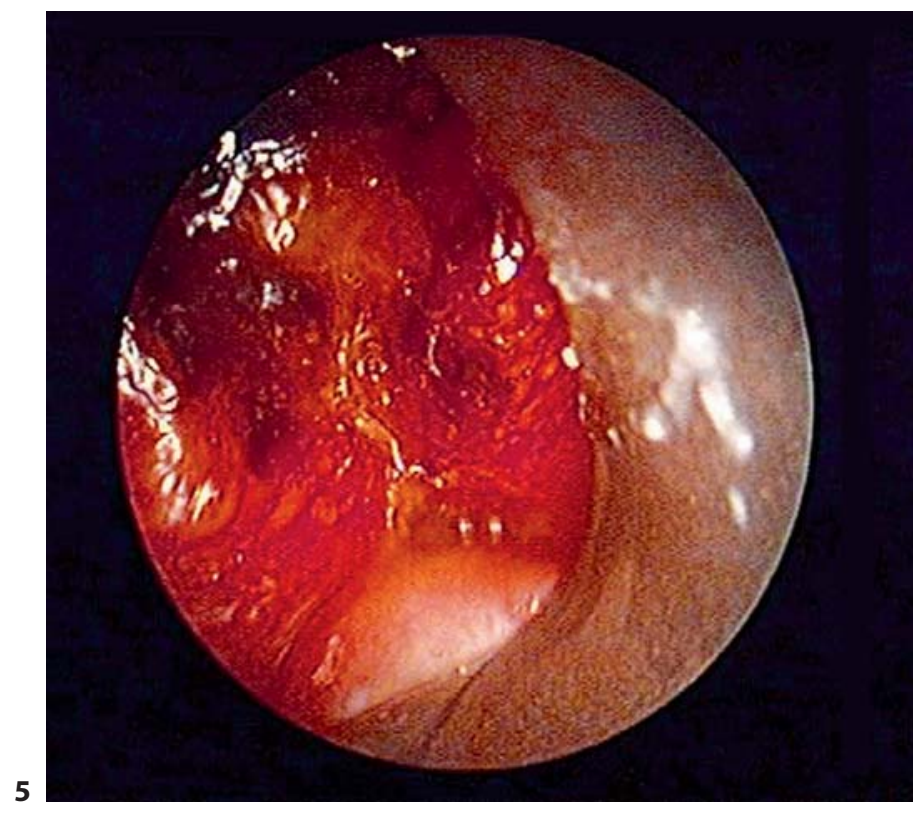

Fig. 5-7. Rigid bronchoscopy: intraluminal tumour mass leading to airway obstruction in the right main bronchus (5). After bronchial debulking by laser therapy (6), a stent (7) is placed to stabilize airway patency.

patients, because EBRT provided better overall symptom palliation. Furthermore, a combination of external beam radiation therapy and HDR brachytherapy seems to provide better symptom control especially in patients with poststenotic atelectasis. HDR brachytherapy alone is only recommended for symptomatic patients with recurrent endobronchial obstruction previously treated by EBRT, providing it is technically feasible.
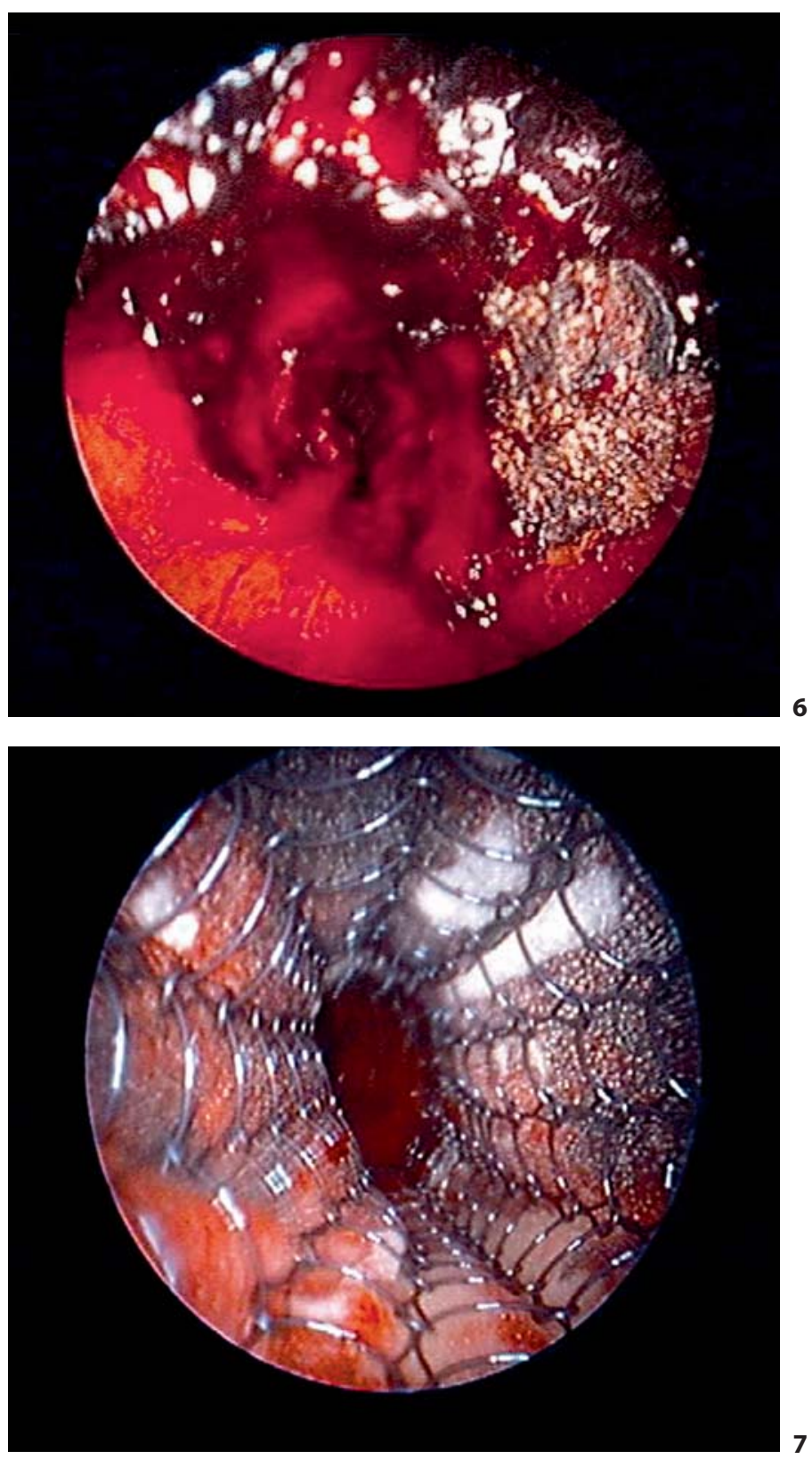

In summary, there are a lot of therapeutic modalities for the treatment of CAO. To achieve a rapid symptom control in a life-threatening situation, several bronchoscopic techniques are available. In most cases, a combination of these endoscopic methods is required (fig. 5-7). Mechanical and thermic endoscopic procedures like laser, APC or cryotherapy provide rapid relief of symptoms due to central airway stenosis. To prevent recurrence of obstruction due to intraluminal tumour growth or to

Gompelmann/Eberhardt/Herth 
treat obstruction due to extraluminal compression, the insertion of a stent provides re-establishment of the patency of obstructed airways. Palliative brachytherapy is another option for CAO treatment, but the effects of brachytherapy are delayed and should only be considered in respiratorily stable patients. Indication for palliative brachytherapy is a CAO in patients who are not able to undergo EBRT or surgery due to a poor performance status or who have a tumour recurrence after receiving surgery or a maximum dose of EBRT.

\section{Tracheo-Oesophageal Fistula}

Tracheo-oesophageal fistula is less common in lung cancer but it is a more common complication in oesophageal cancer. Nevertheless, there are some lung cancer patients presenting a tracheo-oesophageal fistula. Cough, dyspnoea and recurrent pneumonia are the typical features [47]. Due to poor prognosis in locally advanced lung cancer or metastatic disease with survival from weeks to months, surgical intervention offers no advisable option. The goal of therapy consists in the palliation of symptoms by restoring patency of the trachea that can be achieved by stenting. Implantation of an oesophageal tube alone often fails to seal the fistula; furthermore, a protrusion of this tube into the lumen of the airway with consequently compromised ventilation is often observed. Therefore, a simultaneous insertion of an airway stent is necessary to prevent the protrusion of the oesophageal tube and maintain ventilation. Furthermore, the airway stent helps sealing the fistula [33].

\section{Haemoptysis}

Common causes for haemoptysis are lung cancer [48] and inflammatory processes like tuberculosis, bronchiectasis or bronchitis depending on the local circumstances. In Europe, lung cancer is the leading cause for haemoptysis; however, in the developing world, inflammatory diseases are the most common cause [49]. During their clinical course, $20 \%$ of all patients with bronchogenic carcinoma develop haemoptysis [50]. They are more common in NSCLC than in SCLC [51]. The amount of haemoptysis can vary from blood-stained sputum to life-threatening massive bleeding. Massive haemoptysis is defined as the expectoration of $200-1,000 \mathrm{ml}$ blood in a period of $24-48 \mathrm{~h}$ [52]. In $3 \%$ of the lung cancer patients, severe massive bleeding is the cause of death [53]. There- fore, urgent intervention in case of severe haemoptysis is required. There are many different bronchoscopic techniques but other interventional procedures like bronchial arterial embolization or palliative thoracic radiotherapy can also be used.

\section{Endoscopic Management of Haemoptysis}

Bronchoscopy plays not only an important role for diagnosis, but also for the urgent management of severe haemoptysis. Endoscopic methods include cold-saline lavage, instillation of topical vasoconstrictive agents or fibrinogen-thrombin, balloon or stent tamponade, endobronchial airway blockade, laser therapy, APC and electrocautery.

In case of peripheral bleeding, one endoscopic method is the cold-saline lavage. This simple procedure, first reported in 1980, consists of instillation of saline at $4^{\circ} \mathrm{C}$ in the airways leading to a quick stop of bleeding [53]. However, this procedure has often only a short-term effect so that further therapy is required. In mild to moderate bleeding, the endobronchial application of vasoconstrictive agents like epinephrine can be effective [50]. When using epinephrine there is the risk of acute hypertension, pulmonary oedema and arrhythmias due to high plasma levels [54] that should be considered especially in patients with cardiac comorbidity. Other vasoconstrictive agents that can be used for endobronchial instillation are antidiuretic hormone derivatives like ornipressin or terlipressin [55]. The approach of fibrinogen and/or thrombin instillation for bronchoscopic haemostasis has already been reported in the 1980s. This method consists of promotion of endobronchial clot formation by induction of fibrin polymerization. In a study published by Tsukamoto et al. [56] in 1989, thrombin therapy was effective in $74 \%$, and in $100 \%$ a control of bleeding was achieved with a fibrinogen/thrombin combination. Another prospective study, in which 11 patients with severe haemoptysis were enrolled, confirmed the immediate efficacy of fibrinogen-thrombin instillation in $100 \%$. An early relapse of haemoptysis occurred in $18 \%$, a long-term relapse in 9\% [57].

Another endoscopic technique is the endobronchial tamponade by a balloon or a stent that can be performed especially in cases of life-threatening bleeding. Although a balloon tamponade is already an old method that was initially described in 1974, it is still used successfully in case of severe bleeding [50]. In this procedure, the bleeding bronchus is occluded by a balloon that is inflated and kept in place. The balloon tamponade can especially be performed in haemodynamically unstable patients to 
bridge the life-threatening situation until the patient can undergo definitive therapy like surgery. Different bronchoscopic balloon tamponade techniques are described but the therapeutic principle is the same [50]. Occlusive stent placement is an alternative quite novel management approach to severe haemoptysis from lung cancer. In a case report published by Brandes et al. [58] in 2008, an immediate tamponade of massive haemoptysis from a cavitary lung cancer could be achieved by the implantation of 2 covered self-expanding airway stents. The insertion of gauze, gelfoam or an oxidized regenerated cellulose mesh is another technique to achieve tamponade. In 2005, Valipour et al. [59] demonstrated the benefit of bronchoscopy-guided topical haemostatic tamponade therapy using oxidized regenerated cellulose mesh. First, the oxidized regenerated cellulose, a knitted fabric, can be cut to the appropriate size. Then, the mesh is grasped with forceps and then pulled back into the operating channel until it is enclosed in the end of the flexible bronchoscope. Afterwards it can be placed into the bleeding airway under visual control. In 56 out of 57 patients (98\%), immediate arrest of haemoptysis could be achieved. Only in 1 patient haemoptysis could not be controlled by topical haemostatic tamponade. All patients successfully treated with topical haemostatic tamponade remained free of haemoptysis for the first $48 \mathrm{~h}$. Recurrence of haemoptysis occurred in $10.5 \%$ of the patients.

Endobronchial airway blockade by placement of a silicone spigot is another technique that is used preferably in massive life-threatening bleeding allowing stabilizing haemodynamically unstable patients prior to surgical intervention or endovascular embolization. In 2006, Dutau et al. [60] reported the first case of using such a silicone spigot in case of massive haemoptysis to stabilize the patient. The spigot was placed successfully by a flexible bronchoscope and remained in the bronchi during arterial embolization. Afterwards, the silicone spigot could be removed.

When the source of bleeding is bronchoscopically visible, laser coagulation offers an effective treatment option for haemoptysis. Especially in lung cancer patients with endoluminal tumour in the central airway, laser photocoagulation provides a definitive approach to manage haemoptysis due to effective haemostasis but results also in vaporization of the endoluminal tumour mass. Therefore, the indication for laser treatment in lung cancer patients is not only haemoptysis but concomitant CAO. In a retrospective analysis by Han et al. [61], 110 patients with lung cancer were treated with Nd:YAG laser because of dyspnoea, haemoptysis and cough. In 52 patients, hae- moptysis was the leading symptom. After treatment, $77 \%$ of these patients were free of haemoptysis, $17 \%$ of the patients reported subjective improvement of haemoptysis. Only in $6 \%$ of the patients could no improvement be achieved. Similar to laser therapy is the use of APC that can also be used in case of visible bleeding lesions. Haemoptysis and concomitant airway stenosis are the leading indications. The effectiveness in these 2 indications was confirmed in a retrospective study by Morice et al. [62]. Sixty patients with haemoptysis and/or airway obstruction due to lung cancer, metastatic tumours affecting the central bronchi and benign airway diseases were treated by APC. In all patients, immediate arrest of bleeding could be detected. Furthermore, nearly all patients with airway obstruction except 1 experienced improvement of dyspnoea.

\section{Arterial Embolization}

Beside the bronchoscopic techniques, palliative arterial embolization is another option to manage haemoptysis in lung cancer patients. Although the most common indications for arterial embolotherapy are haemoptysis due to benign lung diseases like bronchiectasis, cystic fibrosis or tuberculosis, the embolization offers also an effective therapeutic modality in lung cancer patients. In 2007, Park et al. [63] published the results of a prospective study evaluating the efficacy and safety of arterial embolization in patients with primary lung cancer. Nineteen lung cancer patients presenting haemoptysis underwent arterial embolization. In all patients, the embolization was performed without complications. In $79 \%$ of the patients, clinical success was achieved: in $63 \%$ a complete arrest of haemoptysis was obtained, in $16 \%$ a partial arrest. The overall recurrence rate of haemoptysis was 33\%.

\section{Palliative Thoracic Radiotherapy}

Thoracic radiotherapy is another option to control symptoms that are related to the locoregional tumour growth. Eight studies on the role of radiotherapy in advanced lung cancer summarized in a review published in 2001 showed that in particular haemoptysis and also chest pain can be improved in $60-80 \%$ by palliative thoracic radiotherapy [64]. These results could be confirmed by a recent study including 1,250 patients with locally advanced or metastatic NSCLC [65]. All patients received a short-time, split-course palliative thoracic radiotherapy. In $68 \%$ of all cases, haemoptysis was palliated, but also chest pain, cough, dyspnoea, hoarseness and dysphagia could be improved. In addition, $92 \%$ of all patients tolerated the radiotherapy very well. 


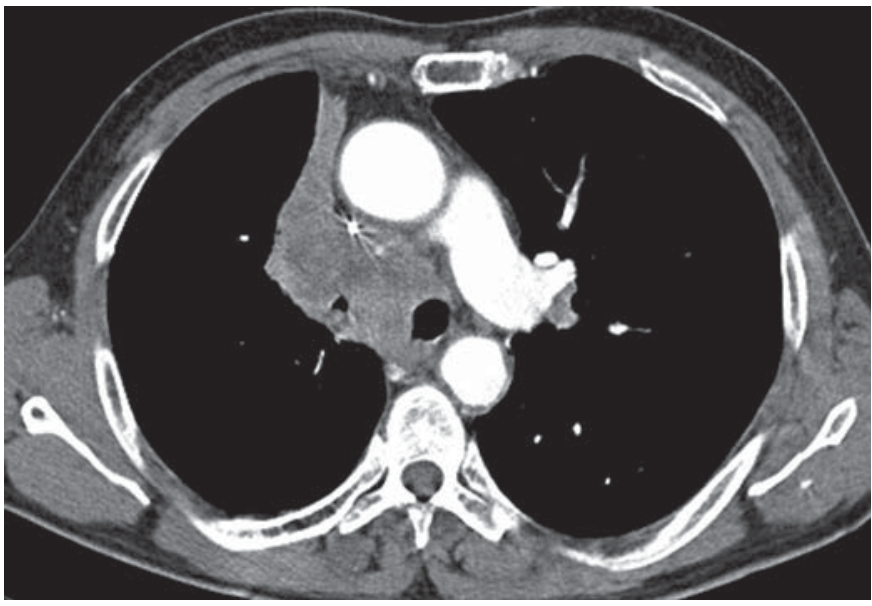

Fig. 8. Contrast-enhanced computed tomography image of a right upper lobe tumour leading to SVCO. Courtesy of C.P. Heussel, Radiology, Thoraxklinik at the University of Heidelberg, Germany.

In summary, there are many different techniques to manage haemoptysis in lung cancer patients. Bronchoscopic interventions as well as arterial embolization or palliative thoracic radiotherapy provide symptom control with subsequent improvement of the patient's quality of life.

\section{SVC Syndrome}

Lung cancer is the most common cause for SVC syndrome [47]. It accounts for $65-80 \%$ of SVC obstruction (SVCO) in reported series [1]. Thereby, tumour mass (fig. 8) or enlarged mediastinal lymph node metastases can lead to compression of the SVC. SVC syndrome is more common in patients with SCLC than with NSCLC. At the time of diagnosis, SVC syndrome can be observed in $10 \%$ of patients with SCLC and in $1.7 \%$ of patients with NSCLC [66]. Swelling of arms, face, eyelids and neck as well as dyspnoea are typical features in case of SVC syndrome. Furthermore, headache due to the local hypertension is often present. Physical examination often reveals dilated neck veins or a prominent venous pattern on the chest. Treatment of SVCO includes SVC stenting, chemotherapy and radiotherapy [47].

\section{SVC Stenting}

To achieve rapid relief of symptoms, SVC stenting is the therapy of choice. Following dilation of the vena cava obstruction with a balloon, the stent is positioned across the obstruction to provide free flow of blood into the right atrium. Thereby, intramural tumour growth or thrombosis are absolute contraindications [67]. In a review [66] including 23 cases related to stent therapy of SVCO, data show a relief of vena cava obstruction in 151 patients out of 195 patients (95\%) with subsequent improvement of SVCO-related symptoms. Following stenting, $10.7 \%$ of the patients experienced an SVCO relapse due to thrombus within the stent or tumour ingrowth at intervals ranging from 3 days to 8 months. In $76.5 \%$ of all patients with relapse, stent patency could be restored by thombolysis or further stent insertion. The effectiveness of SVC stenting in case of obstruction due to malignant tumours was confirmed in several more recently performed studies with similar results [68-70].

\section{Chemotherapy and Radiotherapy}

Chemotherapy as well as radiotherapy can be used in the treatment of SVCO due to lung cancer, whereby the efficacy depends on histology. In SCLC, chemotherapy alone relieved SVCO in $76.9 \%$ and radiotherapy alone in $77.6 \%$. The response rate of SVCO relief in a small number of patients receiving a synchronous chemoradiotherapy was $83.3 \%$ [66]. In NSCLC, the relief of SVCO following chemotherapy or radiotherapy is less convincing. Chemotherapy relieved SVCO in $59.0 \%$ and radiotherapy in $63.0 \%$. The response rate in case of synchronous chemoradiotherapy was only $31.3 \%$ [66].

In conclusion, SVC syndrome is a lung cancer-related complication often requiring urgent intervention. In patients with symptomatic SVCO due to SCLC, a chemotherapy is recommended. In case of chemotherapy failure, a stent insertion should be considered. Stent insertion and/or radiotherapy are the therapeutic pillars in patients with SVCO due to NSCLC [47].

\section{References}

Respiration 2011;82:111-123
1 Midthun DE, Jett JR: Lung tumors; in Albert RK, Spiro SG, Jett JR (eds): Clinical Respiratory Medicine, ed 3. Philadelphia, Mosby Elsevier, 2008, pp 605-632.

2 Jackman DM, Johnson BE: Small cell lung cancer. Lancet 2005;366:1385-1396.

- 3 Goeckenjan G, Sitter H, Thomas M, et al: Prevention, diagnosis, therapy and followup of lung cancer. Pneumologie 2010;64(suppl 2):e1-e164.

$\checkmark 4$ Khaleeq G, Musani A I: Emerging paradigms in the management of malignant pleural effusion. Respir Med 2008;102:939948. 
5 Neragi-Miandoab S: Surgical and other invasive approaches to recurrent pleural effusion with malignant etiology. Support Care Cancer 2008;16:1323-1331.

6 Loddenkemper R, Mathur PN, Noppen M, Lee P: Indications for and results of medical thoracoscopy; in Loddenkemper R, Mathur PN, Noppen M, Lee P (eds): Medical Thoracoscopy/Pleuroscopy. Stuttgart, Thieme, 2010, pp 22-52.

7 Aydogmus U, Ozdemir S, Cansever L, Sonmezoglu Y, Kocaturk Cl, Bedirhan MA: Bedside talc pleurodesis for malignant pleural effusion: factors affecting success. Ann Surg Oncol 2009;16:740-750.

$\checkmark 8$ Dresler CM, Olak J, Herndon JE 2nd, Richards WG, Scalzetti E, Fleishman SB, Kernstine KH, Demmy T, Jablons DM, Kohman L, Daniel TM, Haasler GB, Sugarbaker DJ: Phase III intergroup study of talc poudrage vs talc slurry sclerosis for malignant pleural effusion. Chest 2005;127:909-915.

-9 Stefani A, Natali P, Casali C, Morandi U: Talc poudrage versus talc slurry in the treatment of malignant pleural effusion: a prospective comparative study. Eur J Cardiothorac Surg 2006;30:827-832.

10 Tan C, Sedrakyan A, Browne J, Swift S, Treasure T: The evidence on the effectiveness of management for malignant pleural effusion: a systematic review. Eur J Cardiothorac Surg 2006;29:829-838.

-11 Sioris T, Sihvo E, Salo J, Räsänen J, Knuuttila A: Long-term indwelling catheter (PleurX) for malignant pleural effusion unsuitable for talc pleurodesis. Eur J Surg Oncol 2009;35: 546-551.

-12 Musch E, Gremmler B, Nitsch J, Rieger J, Malek M, Chrissafidou A: Intrapericardial instillation of mitoxantrone in palliative therapy of malignant pericardial effusion. Onkologie 2003;26:135-139.

13 Lestuzzi C: Neoplastic pericardial disease: old and current strategies for diagnosis and management. World J Cardiol 2010;2:270279.

14 Vaitkus PT, Herrmann H, Le Winter M: Treatment of malignant pericardial effusion. JAMA 1994;272:59-64.

-15 Marcy PY, Bondiau PY, Brunner P: Percutaneous treatment in patients presenting with malignant cardiac tamponade. Eur Radiol 2005;15:2000-2009.

-16 Kopecky SL, Callahan JA, Tajik AJ, Seward JB: Percutaneous pericardial catheter drainage: report of 42 consecutive cases. Am J Cardiol 1986;58:633-635.

-17 Swanson N, Mirza I, Wijesinghe N, Devlin G: Primary percutaneous balloon pericardiotomy for malignant pericardial effusion. Catheter Cardiovasc Intervent 2008;71:504507.

$\checkmark 18$ Shepherd FA, Morgan C, Evans WK, et al: Medical management of malignant pericardial effusion by tetracycline sclerosis. Am J Cardiol 1987;60:1161-1166.
19 Maher EA, Shepherd FA, Todd TJ: Pericardial sclerosis as the primary management of malignant pericardial effusion and cardiac tamponade. J Thorac Cardiovasc Surg 1996; 112:637-643.

20 Liu G, Crump M, Goss PE, Dancey J, Shepherd FA: Prospective comparison of the sclerosing agents doxycycline and bleomycin for the primary management of malignant pericardial effusion and cardiac tamponade. J Clin Oncol 1996;14:3141-3147

21 Kunitoh H, Tamura T, Shibata T, Imai M, Nishiwaki Y, Nishio M, Yokoyama A, Watanabe K, Noda K, Saijo N; JCOG Lung Cancer Study Group, Tokyo, Japan: A randomised trial of intrapericardial bleomycin for malignant pericardial effusion with lung cancer (JCOG9811). Br J Cancer 2009;100:464-469.

22 Moriya T, Takiguchi Y, Tabeta H, Watanabe R, Kimura H, Nagao K, Kuriyama T: Controlling malignant pericardial effusion by intrapericardial carboplatin administration in patients with primary non-small-cell lung cancer. Br J Cancer 2000;83:858-862.

23 Tomkowski WZ, Filipecki S: Intrapericardial cisplatin for the management of patients with large malignant pericardial effusion in the course of the lung cancer. Lung Cancer 1997; 16:215-222.

24 Breitenbücher A, Chhajed PN, Brutsche MH, Mordasini C, Schilter D, Tamm M: Longterm follow-up and survival after Ultraflex stent insertion in the management of complex malignant airway stenoses. Respiration 2008;75:443-449.

25 Seijo LM, Sterman DH: Interventional pulmonology. N Engl J Med 2001;344:740-749.

26 Dutau H, Breen DP: Endobronchial laser treatment: an essential tool in therapeutic bronchoscopy; in Strausz J, Bolliger CT (eds): Interventional Pulmonology. European Respiratoy Monograph 48. Sheffield, European Respiratory Society, 2010, pp 149-160.

27 Cavaliere S, Venuta F, Foccoli P, Toninelli C, La Face B: Endoscopic treatment of malignant airway obstructions in 2,008 patients. Chest 1996;110:1536-1442.

28 Zaric B, Canak V, Milonvancev A, Jovanovic S, Budisin E, Sarcev T, Nisevic V: The effect of Nd:YAG laser in resection on symptom control, time to progression and survival in lung cancer patients. J Buon 2007;12:361368 .

29 Morgan RK, Ernst A: Cryosurgery; in Strausz J, Bolliger CT (eds): Interventional Pulmonology. European Respiratoy Monograph 48. Sheffield, European Respiratory Society, 2010, pp 161-172.

30 Vachani A, Sterman HD: Bronchoscopy; in Albert RK, Spiro SG, Jett JR (eds): Clinical Respiratory Medicine, ed 3. Philadelphia, Mosby Elsevier, 2008, pp 177-196.

31 Morice RC, Ece T, Ece F, Keus L: Endobronchial argon plasma coagulation for treatment of hemoptysis and neoplastic airway obstruction. Chest 2001;119:781-787.
32 Schumann C, Hetzel M, Babiak AJ, Hetzel J, Merk T, Wibmer T, Lepper PM, Krüger S: Endobronchial tumor debulking with a flexible cryoprobe for immediate treatment of malignant stenosis. J Thorac Cardiovasc Surg 2010;139:997-1000.

33 Freitag L: Airway stents; in Strausz J, Bolliger CT (eds): Interventional Pulmonology. European Respiratoy Monograph 48. Sheffield, European Respiratory Society, 2010, pp 190 217.

34 Bolliger CT, Probst R, Tschopp K, Solé M, Perruchoud AP: Silicone stents in the management of inoperable tracheobronchial stenoses: indications and limitations. Chest 1993;104:1653-1659.

35 Puma F, Ragusa M, Avenia N, Urbani M, Droghetti A, Daddi N, Daddi G: The role of silicone stents in the treatment of cicatricial tracheal stenoses. J Thorac Cardiovasc Surg 2000;120:1064-1069.

- 36 Miyazawa T, Yamakido M, Ikeda S, Furukawa K, Takiguchi Y, Tada H, Shirakusa T: Implantation of Ultraflex nitinol stents in malignant tracheobronchial stenoses. Chest 2000;118:959-965.

37 Oki M, Saka H, Kitagawa C, Kogure Y: Silicone Y-stent placement on the carina between bronchus to the right upper lobe and bronchus intermedius. Ann Thorax Surg 2009;87:971-974.

38 Dutau H, Toutblanc B, Lamb C, Seijo L: Use of the Dumon Y-stent in the management of malignant disease involving the carina: a retrospective review of 86 patients. Chest 2004;126:951-958.

39 Freitag L, Tekolf E, Stamatis G, Greschuchna D: Clinical evaluation of a new bifurcated dynamic airway stent: a 5-year experience with 135 patients. Thorac Cardiovasc Surgeon 1997;45:6-12.

-40 Yang RM, Han XW, Wu G, Li YD, Li FB: Implantation of a self-expandable metallic inverted Y-stent to treat tracheobronchial stenosis in the carinal region: initial clinical experience. Clin Radiol 2007;62:1223-1228.

41 Agrafiotis M, Siempos II, Falagas ME: Infections related to airway stenting: a systematic review. Respiration 2009;78:69-74.

42 Michailidou I, Becker HD, Eberhardt R: Bronchoscopic high-dose-rate brachytherapy; in Strausz J, Bolliger CT (eds): Interventional Pulmonology. European Respiratoy Monograph 48. Sheffield, European Respiratory Society, 2010, pp 173-189.

43 Barber P, Stout R: High dose rate endobronchial brachytherapy for the treatment of lung cancer: current status and indications. Tho$\operatorname{rax}$ 1996;51:345-347.

44 Macha HN, Freitag L: The role of brachytherapy in the treatment and control of central bronchial carcinoma. Monaldi Arch Chest Dis 1996;51:325-328. 
-45 Aumont-le Guilcher M, Prevost B, Sunyach MP, Peiffert D, Maignon P, Thomas L, Williaume D, Beque, Lerouge D, Campion L, Mahe MA: High-dose-rate brachytherapy for non-small-cell lung carcinoma: a retrospective study of 226 patients. Int J Radiat Oncol Biol Phys 2011:79:1112-1116.

46 Ung YC, Yu E, Falkson C, Hayned AE, StysNorman D, Evans WK: The role of highdose-rate brachytherapy in the palliation of symptoms in patients with non-small-cell lung cancer: a systematic review. Brachytherapy 2006;5:189-202.

47 Kvale P A, Selecky PA, Prakash UBS: Palliative care in lung cancer: ACCP evidencebased clinical practice guidelines (2nd edition). Chest 2007;132:368-403.

$\checkmark 48$ Fidan A, Ozdogan S, Oruc O, Salepci B, Ocal Z, Caglayan B: Hemoptysis: a retrospective analysis of 108 cases. Respir Med 2002;96: 677-680.

49 Kreit JW: Hemoptysis; in Albert RK, Spiro SG, Jett JR (eds): Clinical Respiratory Medicine, ed 3. Philadelphia, Mosby Elsevier, 2008, pp 657-665.

50 Sakr L, Dutau H: Massive hemoptysis: an update on the role of bronchoscopy in diagnosis and management. Respiration 2010;80:3858.

51 Salajka F: Occurrence of haemoptysis in patients with newly diagnosed lung malignancy. Schweiz Med Wochenschr 1999;129: 1487-1491.

52 Jones DK, Davies RJ: Massive hemoptysis. BMJ 1990;300:889-890.

53 Conlan AA, Hurwitz SS: Management of massive haemoptysis with the rigid bronchoscope and cold saline lavage. Thorax 1980; 35:901-904.
Kalyanaraman M, Carpenter RL, McGlew MJ, Guertin SR: Cardiopulmonary compromise after use of topical and submucosal $\alpha$ agonists: possible added complication by the use of $\beta$-blocker therapy. Otolaryngol Head Neck Surg 1997;117:56-61.

55 Tuller C, Tuller D, Tamm M, Brutsche MH: Hemodynamic effects of endobronchial application of ornipressin versus terlipressin. Respiration 2004;71:397-401.

56 Tsukamoto T, Sasaki H, Nakamura H: Treatment of hemoptysis patients by thrombin and fibrinogen-thrombin infusion therapy using a fiberoptic bronchoscope. Chest 1989; 96:473-476.

-57 De Gracia J, de la Rosa D, Catalán E, Alvarez A, Bravo C, Morell F: Use of endoscopic fibrinogen-thrombin in the treatment of severe hemoptysis. Respir Med 2003;97:790795.

58 Brandes JC, Schmidt E, Yung R: Occlusive endobronchial stent placement as a novel management approach to massive hemoptysis from lung cancer. J Thorac Oncol 2008;3: 1071-1072.

59 Valipour A, Kreuzer A, Koller H, Koessler W, Burghuber OC: Bronchoscopy-guided topical hemostatic tamponade therapy for the management of life-threatening hemoptysis. Chest 2005;127:2113-2118.

60 Dutau H, Palot A, Haas A, Decamps I, Durieux O: Endobronchial embolization with a silicone spigot as a temporary treatment for massive hemoptysis: a new bronchoscopic approach of the disease. Respiration 2006;73: 830-832.

61 Han CC, Prasetyo D, Wright GM: Endobronchial palliation using Nd-YAG laser is associated with improved survival when combined with multimodal adjuvant treatments. J Thorac Oncol 2007;2:59-64.

62 Morice RC, Ece T, Ece F, Keus L: Endobronchial argon plasma coagulation for treatment of hemoptysis and neoplastic airway obstruction. Chest 2001;119:781-787.
63 Park HS, Kim YI, Kim HY, Zo JI, Lee JH, LEE JS: Bronchial artery and systemic embolization in the management of primary lung cancer patients with hemoptysis. Cardiovasc Intervent Radiol 2007;30:638-643.

64 Numico G, Russi E, Marlano M: Best supportive care in non-small cell lung cancer: is there a role for radiotherapy and chemotherapy? Lung Cancer 2001;32:213-226.

65 Reinfuss M, Mucha-Malecka A, Walasek T, Blecharz P, Jakubowicz J, Skotnicki P, Kowalska T: Palliative thoracic radiotherapy in non-small cell lung cancer: an analysis of 1,250 patients. Palliation of symptoms, tolerance and toxicity. Lung Cancer 2011;71:344349

66 Rowell NP, Gleeson FV: Steroids, radiotherapy, chemotherapy and stents for superior vena caval obstruction in carcinoma of the bronchus: a systematic review. Clin Oncol (R Coll Radiol) 2002;14:338-351.

67 Padley SPG, Hansell DM: Imaging techniques; in Albert RK, Spiro SG, Jett JR (eds): Clinical Respiratory Medicine, ed 3. Philadelphia, Mosby Elsevier, 2008, pp 1-68.

68 Greillier L, Barlésa F, Doddoli C, Durieux O, Torre JP, Gimenez C, Kleisbauer JP: Vascular stenting for palliation of superior vena cava obstruction in non-small-cell lung cancer patients: a future 'standard' procedure? Respiration 2004;71:178-183.

69 Nagat T, Makutani S, Uchida H, Kichikawa K, Maeda M, Yoshioka T, Anai H, Sakaguchi $\mathrm{H}$, Yoshimura H: Follow-up results of $171 \mathrm{pa}-$ tients undergoing metallic stent placement for the treatment of a malignant obstruction of the superior vena cava. Cardiovasc Intervent Radiol 2007;30:959-967.

70 Berna P, Bagan P, Renard C, Auguier M, Remond A, Riquet M: Pulmonary malignant superior vena cava obstruction: endovascular stent therapy. Rev Pneumol Clin 2008;64: 129-132. 